\title{
Political competition within and between parties: an application to environmental policy ${ }^{1}$
}

\author{
Helmuth Cremer \\ Toulouse School of Economics \\ (IDEI, GREMAQ and Institut universitaire de France) \\ Philippe De Donder \\ Toulouse School of Economics \\ (IDEI and GREMAQ-CNRS) \\ Firouz Gahvari \\ Department of Economics \\ University of Illinois at Urbana-Champaign
}

August 2005

This revision, July 2007

\footnotetext{
${ }^{1}$ We thank the participants at seminars in Yale, WZB (Berlin), the 2005 PET Conference (Marseille), the Conference in tribute to JJ Laffont (Toulouse), and particularly Stephen Coate, Torsten Persson and John Roemer, for their helpful comments and suggestions. We are particularly grateful to two anonymous referees of this Journal and the Co-Editor, Antonio Merlo, for their useful comments and advice.
} 


\begin{abstract}
This paper presents a political economy model that is consistent with the low rate of emission taxes in the U.S., as well as the fact that neither Democrats nor Republicans propose to increase them. The voters differ according to their wage and capital incomes. They vote over the emission tax rate and a budgetary rule that specifies how to redistribute the tax proceeds. The political competition is modeled à la Roemer (2001) where the two parties care for the policies they propose as well as the probability of winning; the equilibrium solution concept is the Party Unanimity Nash Equilibrium (PUNE). We calibrate the model using U.S. data and compute the PUNEs numerically. Two types of PUNEs emerge. In one, reflecting the preferences of the militants in the two parties, equilibrium is characterized by both parties proposing a very huge tax rate, with Democrats typically beating the Republicans. In the other, dictated more by the concerns of the opportunists who care most about winning elections, both offer subsidies and both have a chance to win the election.
\end{abstract}

Key words: Emission taxes, political competition, PUNE, distributional concerns, political compromise.

JEL Classification: H23, D72. 


\section{Introduction}

The U.S. environmental policy is often criticized by the environmentalists, academics and the media for being exceedingly lax on polluters. The criticism has been particularly acute in relation to the greenhouse gases that burning fossil fuels emit. Two facts are often cited to underline this. The first is that gasoline prices are, and have traditionally been, markedly lower in the U.S. than in any other industrial country. ${ }^{1}$ The second is the U.S. government's refusal to join the more than 140 countries of the world who have ratified the Kyoto protocol. ${ }^{2}$ With no policy change in the offing, one suspects that there must exist strong political pressures pushing the US government to adhere to its current policies. This is all the more likely as the issue is not one of Democrats versus Republicans. Neither party supports higher gasoline taxes, nor ratifying the Kyoto protocol.

This paper presents a political economy model that is consistent with the persistence of the U.S. environmental policy, as well as the fact that neither party offers a policy proposal of consequence for changing it. We model the political competition à la Roemer (2001) with the "Party Unanimity Nash Equilibrium" (PUNE) as the equilibrium solution concept. This approach is a more realistic, and historically a more accurate, description of the political process, as compared to the traditional approach which explains every political outcome through the prism of a median voter's preferences. As Roemer (2001) has forcefully argued, the traditional approach suffers from three major shortcomings. First, its Downsian outlook, wherein the political parties (as opposed to voters) have no preferences over policies, accords political parties no role in determining the outcome of the political process within a society. This is plainly counterfactual. Second, the assumption that the distribution of voter types is known for certain, is rather dubious. Third, there is the well-known problem of the generic nonexistence of majority-voting equilibrium when the policy space is multidimensional.

In Roemer's approach, two political parties (here Democrats and Republicans) compete for votes in an election. Each chooses a policy that it will implement if elected.

\footnotetext{
${ }^{1}$ Currently, a litre of gasoline is taxed about 10 cents in the U.S., compared to 96 cents in France and 1.10 dollar in the UK.

${ }^{2}$ Australia is the only other industrial country not having signed the Kyoto protocol.
} 
Parties are composed of two factions: the "opportunists" who aim to maximize the probability of winning the election, and the "militants" who are only interested in policies regardless of their electoral ramifications. Unanimity between the two factions is required for a party to accept a deviation from its current policy. This unanimity rule determines the preferences (payoffs) of the two parties who simultaneously choose their political platforms. A PUNE is a Nash equilibrium of this game.

We consider a two-dimensional policy comprising a tax (or a subsidy) and a "budgetary rule". The tax (or subsidy) is levied on the consumption of an externality generating (polluting) good; the budgetary rule specifies the way the tax proceeds are redistributed to the polity (or the way the subsidy will have to be financed). Individuals have identical Gorman-polar form preferences over a (non-polluting) numeraire good, the polluting good, and the total level of emissions. The choice of Gorman-polar preferences is consistent with the empirical evidence for the income elasticity of such goods. Poterba (1991) has estimated that, with very few exceptions, the expenditure shares of such polluting goods as gasoline, fuel oil, natural gas, and electricity decrease at all income deciles as income increases. The goods are produced by a linear technology subject to constant returns to scale in a competitive environment. This is essentially the setup we used in our earlier study; see Cremer et al. (2004a). However, that study used a hybrid normative/positive approach and did not use actual empirical data to calibrate the tax/subsidy rates. ${ }^{3}$

We calibrate our model using data for the U.S. economy. The voters are US house-

\footnotetext{
${ }^{3}$ Cremer et al. (2004a) assumed a unidimensional voting game over emission taxes only. We left the disbursement of the tax revenues to be determined at a "constitutional level," by a "welfare maximizing" government. This led us to assert that the government should be able (under certain conditions) to effect first-best Pigouvian emission taxes.

In Cremer et al. (2004b), we followed a positive approach throughout; but, faced with the nonexistence problem when voting simultaneously on two dimensions, we resorted to two sequential voting procedures (with either policy being determined first and the other later), as well as the Shepsle procedure, to arrive at a political equilibrium. Restricting preferences to be quasi-linear, we found that (in most cases) the equilibrium corresponded to the preferences of the "median individual". This included the prediction that all tax revenues must be rebated solely through either wage subsidies or capital income subsidies. Actual policies, of course, never display such a knife-edge property.

Most recently, in Cremer et al. (2007) we revert back to models of unidimensional voting games over emission taxes (leaving the determination of the disbursement of the tax revenues totally out). This paper also uses actual data and the PUNE concept but in a one-dimensional framework. Additionally, it studies the implications of the majority-voting and probabilistic-voting approaches for the equilibrium tax rate.
} 
holds as represented by the 2001 Panel Study for Income Dynamics Survey, considering the 6,877 households who reported a nonzero income (whether labor or asset incomes) for the year 2000. We then fit a bivariate lognormal distribution for labor and asset incomes to this truncated sample (using the same weights that the survey assigns to each household.)

The polluting good is called energy and consists of an aggregate of energy-related consumption goods (fuel oil, gasoline, natural gas, kerosene, LPG and electricity). We calibrate the demand function on the basis of a -0.30 price elasticity of demand (based on the literature estimates for the price elasticity of consumer demand for energy), a marginal propensity to consume of $2.25 \%$ and a 0.0555 ratio of average expenditure on energy to average income (these last two based on the Consumer Expenditure Surveys, 2002), with the average income being determined from the 2001 PSID data $(\$ 59,926)$. The quantity of pollution is found according to the carbon content of each, appropriately-weighted, component. Using a value of $\$ 50$ for the social marginal cost of a ton of carbon (based on the estimates reported by the EPA), we are able to calculate the marginal social damage of one unit of the polluting good. Finally, we find the weights that the two parties' militants (the Democrats and the Republicans in our setting) assign to different citizens, and the probability that a particular citizen participates in the election, on the basis of Bartels (2002). Both weights (for both parties' militants) and turn out probabilities increase with citizens' total income.

We show that the PUNEs that emerge may be grouped into two different types. Type I PUNEs are characterized by both parties proposing very huge tax rates (121\% to $123 \%$ in addition to the current taxes) combined with a budgetary rule which calls for all tax proceeds to be rebated solely on the basis of the voters' capital incomes. These tax rates correspond to the most-preferred tax rates of the militants in the Democratic and the Republican parties. They are extremely high, considering that the optimal unweighted utilitarian policy calls for a tax of only about $10 \%$, and that a person with the median wage and the median capital income prefers the current energy prices to be cut by as much as $89.8 \%$. Thus these PUNEs are essentially dictated by the preferences of the militants in the Democratic and the Republican parties, and especially by the fact that militants in both parties put higher weights on richer individuals' preferences. 
An interesting feature of these PUNEs is that they lead to a Democratic victory with an average probability of (among all PUNEs) 91\% (ranging from 90.8\% to 91.2\%).

Type II PUNEs share the following characteristics. First, both parties offer a subsidy with respect to the current prices. Specifically, the Democrats offer a subsidy that varies from $0.5 \%$ to $70.9 \%$ across all PUNEs (with an average of $24.9 \%$ ), while the Republicans offer a subsidy varying from $0.8 \%$ to $71.6 \%$ (with an average of $30.9 \%$ ). That all Type II PUNEs entail subsidies, underscores the importance of distributional concerns in ensuring political support for environmental policies - a factor that the literature on environmental taxation has, with few exceptions, ignored. Second, both parties offer an interior solution for the budgetary rule despite the fact that all voters have extreme preferences (they want all rebates or taxes to be linked solely either to capital incomes or to wage incomes). This compromise turns out to be a particularly striking feature of the PUNEs; other equilibrium concepts, even when they are not empty in multidimensional choice sets, do not share this feature; see Cremer et al. (2004a). It underlines the importance of generating political support for environmental policies (through political compromise) - another aspect that the literature has hitherto paid scant attention to. ${ }^{4}$

Type II PUNES thus appear to be more consistent with the making of environmental tax policy in the US; namely, the observed reluctance of politicians to impose substantial energy taxes. Additionally, these PUNEs have another feature consistent with reality not present in Type I PUNEs. Under them, Democrats win with an average probability of (among all PUNEs) 54.1\% (ranging from 48.9\% to 69.6\%), and the Republicans win with an average probability of $45.9 \%$ (30.4\% to $51.1 \%$ ). These probabilities are, intuitively, comforting. At $54.1 \%$ and $45.9 \%$, they are rather close. Moreover, the maximum winning probabilities for the two parties over the set of Type II PUNEs exceed, while their minimum winning probabilities fall below, the $50 \%$ mark. The US environmental policy has thus been dictated more by the concerns of the opportunists who care most about winning elections. Nevertheless, the existence of Type I PUNEs suggests the possibility of a change in this policy and arriving at an equilibrium with the militants at the helm. Finally simulations reveal that the same two type of PUNEs,

\footnotetext{
${ }^{4}$ Exceptions include Boyer and Laffont (1998), Bös (2000), Brett and Keen (2000), Marsiliani and Renström (2000), and Cremer, De Donder and Gahvari (2004a, 2004b, 2007).
} 
with the exact same properties, emerge under different income elasticities of demand for polluting goods, as well as when one allows for the existing income taxes to be distortionary. Our results appear to be quite robust.

\section{The model}

Individuals are identified by the "type parameter" $\theta=(r, w)$, where $r$ is capital income and $w$ is labor income. Let $H$ denote the type space; $\theta$ is continuously distributed over $H$ according to the density function, $f(\theta)$. The associated cumulative distribution function is $F(\theta)$. Population size is normalized at one. Total income is $m(\theta)=r+w$. All sources of income are exogenous. Individuals have identical Gorman-polar form preferences over a numeraire good (non-polluting), a polluting good, $y$, and the total level of emissions due to it, $Y$.

The goods are produced by a linear technology subject to constant returns to scale in a competitive environment. Normalize the producer price of $y$ at one. Let $q$ denote the consumer price of $y, I(\theta)$ the disposable income (net of taxes or transfers) and $Y$ the total consumption of $y$ (across all individuals). ${ }^{5}$ The indirect utility function of an individual of type $\theta$ is given by:

$$
v(q, I, Y)=a(q)+b(q) I(\theta)-\varphi(Y)
$$

where $a(q)$ is thrice, and $b(q)$ and $\varphi(Y)$ are twice, continuously differentiable with $a^{\prime}(q) \leq 0, b^{\prime}(q) \leq 0, \varphi^{\prime}(Y)>0$, and $\varphi^{\prime \prime}(Y) \geq 0$. Observe that, ignoring the externality term, $b^{\prime}(q) \equiv 0$ yields a quasi-linear specification; on the other hand, $a(q) \equiv 0$ represents the case of homothetic preferences. By Roy's identity, the demand for $y$ is given by ${ }^{6}$

$$
y(q ; \theta)=-\frac{\partial v / \partial q}{\partial v / \partial I}=c(q)+d(q) I(\theta)
$$

where

$$
c(q)=\frac{-a^{\prime}(q)}{b(q)}>0, \quad \text { and } \quad d(q)=\frac{-b^{\prime}(q)}{b(q)}>0 .
$$

\footnotetext{
${ }^{5}$ The units of measurements are chosen such that one unit of $y$ produces one unit of emissions.

${ }^{6} \mathrm{We}$ are assuming that the consumer has some exogenous income so that his demand for $y$ remains positive even if $I(\theta)=0$.
} 
Aggregate consumption of the polluting good is then equal to

$$
Y=\int_{H} y(q) f(\theta) d \theta=\bar{y}(q)
$$

so that total and average consumption levels are all equal. Observe that the variation in a single individual's consumption of $y$ will have no impact on $Y$.

The existing tax structure consists of labor and capital income taxes. Good $y$ is to be subjected to a "pollution tax" levied at the rate of $(q-1)$ per unit of output. The proceeds of the tax are refunded through reductions in labor and capital income taxes. We do not a priori restrict it to be positive. Consequently, negative "refunds" are not ruled out either. To simplify notation, we do not include pre-existing income taxes explicitly. This implies that the income of individual $\theta$ is given by

$$
I(\theta)=\left(1+g_{r}\right) r+\left(1+g_{w}\right) w,
$$

where $g_{r}$ and $g_{w}$ are the refund rates on capital and wage incomes.

Let $\bar{r}, \bar{w}$ and $\bar{m}$ denote average capital income, average labor income and average income:

$$
\bar{r}=\int_{H} r(\theta) f(\theta) d \theta, \quad \bar{w}=\int_{H} w(\theta) f(\theta) d \theta, \quad \bar{m}=\int_{H} m(\theta) f(\theta) d \theta .
$$

The tax and refund rates are related through the government's budget constraint

$$
R(q)=g_{r} \bar{r}+g_{w} \bar{w}
$$

where $R(q)$ is the net revenue raised from taxing the polluting good. Observe that, in light of (5), the government has only two degrees of freedom in choosing its policy instruments. Once $q$ and, say, $g_{r}$ are set, $g_{w}$ is automatically determined. To represent this in a more symmetric way, and to characterize the refund/funding system through a single parameter, we introduce the concept of a "budgetary rule". This specifies the proportion of tax proceeds, $\alpha$, that must be refunded on the basis of wage incomes (alternatively, the proportion of the subsidy cost financed by taxing wage incomes). Formally, $\alpha$ is defined such that ${ }^{7}$

$$
\alpha=\frac{g_{w} \bar{w}}{R(q)}=1-\frac{g_{r} \bar{r}}{R(q)} .
$$

\footnotetext{
${ }^{7}$ If $R(q)=0$, then $g_{r}=g_{w}=0$ as we restrict $g_{r}$ and $g_{w}$ to be of the same sign. In this case, $\alpha=$ $[0,1]$.
} 
With this notation, the tax-cum-refund policy is characterized fully by the pollution tax (or the consumer price of $y$ ) and by the budgetary rule; i.e. by the two parameters $q$ and $\alpha$. Assume that $\alpha \in[0,1]$; this amounts to assuming that $g_{w}$ and $g_{r}$ are restricted to be of the same sign.

\subsection{Existing distortionary taxes}

We do not explicitly model the existing capital and labor income taxes. However, we account for their distortionary nature. Specifically, let $\eta$ denote the marginal cost of public funds. An extra dollar of tax revenue raised through the pollution tax is matched by a refund of $1+\eta$ dollars in order to keep the government's budget constraint balanced. This reflects the fact that lowering the existing tax rates lowers the welfare loss associated with the current tax structure. The argument is symmetric and holds for a subsidy on the polluting good. In this case, funding a one-dollar subsidy on polluting goods through income taxes, costs the treasury $1+\eta$ dollars. ${ }^{8}$ Consequently, we have,

$$
R(q)=(1+\eta)(q-1) Y=(1+\eta)(q-1) \bar{y}(q),
$$

so that $R(q)$ is the revenue raised from taxing the polluting good plus gains due to the reduction in the existing excess burden of income taxes [when they are cut by $(q-1) \bar{y}(q)$ dollars]. Alternatively, for a subsidy on polluting goods, $R(q)$ represents its net budgetary cost.

The determination of $q$ and $\alpha$ through the political process forms the core of our study. The process is one of competition between two parties. However, we depart from the traditional Downsian approach and instead use John Roemer's "Party Unanimity Nash Equilibrium" (PUNE) as our solution concept. We will discuss this solution concept briefly in Section 5 below. The next section examines the voters' preferences over $(q, \alpha)$ and gives a characterization for the "optimal" solution of $(q, \alpha)$. These will provide the other ingredients for our study.

\footnotetext{
${ }^{8}$ The tax recycling issue has played a central role in the double-dividend literature. There are other factors, e.g. the tax interaction effect, that bear on the size of any potential double dividend; see Gahvari (2002). For our purposes, one can think of $\eta$ as denoting the net effect of these different factors.
} 


\section{Voters' preferences over $(q, \alpha)$ and the "optimal" policy}

A voter's preferences over tax and refund policies is derived by incorporating the government's budget constraint (5) into his utility function (1). As a first step, substitute for $g_{w}$ and $g_{r}$ from equation (6) into (4). This yields

$$
I(\alpha, \delta, \theta)=m(\theta)+\delta(\alpha, \theta) R(q),
$$

where

$$
\delta(\alpha, \theta) \equiv(1-\alpha) \frac{r}{\bar{r}}+\alpha \frac{w}{\bar{w}} .
$$

Observe that when the polluting good is taxed, $\delta(\alpha, \theta)$ indicates the proportion of $\theta$ 's tax payment that he will get back in refunds. In the case of a subsidy, $\delta(\alpha, \theta)$ shows the ratio of $\theta$ 's income tax payments to the (price) subsidy he receives from the consumption of the polluting good. ${ }^{9}$ Next, substituting for $I$ from (8) into equation (1), one arrives at the voter's reduced indirect utility function,

$$
V(q, \alpha, \theta)=a(q)+b(q)[m(\theta)+\delta(\alpha, \theta) R(q)]-\varphi(\bar{y}(q)) .
$$

It is clear from (10) that the size of $\delta(\alpha, \theta)$ is a crucial determinant of the impact of $q$ on $V(q, \alpha, \theta)$. Additionally, $\delta(\alpha, \theta)$ is the only direct channel through which $\alpha$ affects $V(q, \alpha, \theta)$.

\subsection{Optimal policy}

To characterize the optimal solution for the pair of policy instruments $(q, \alpha)$, we resort to a utilitarian framework. This provides a natural benchmark against which to assess the properties of our political solution. The utilitarian social welfare function can be written, using (10) and (9), as

$$
W=\int_{H} V(q, \alpha, \theta) f(\theta) d \theta=a(q)+b(q)(\bar{m}+R(q))-\varphi(\bar{y}(q)) .
$$

\footnotetext{
${ }^{9}$ To see this, substitute for $\alpha$ from (6) into (9). This yields

$$
\delta(\alpha, \theta)=\frac{g_{r} r(\theta)+g_{w} w(\theta)}{R(q)} .
$$
}


Observe that $W$ is independent of $\alpha$ so that distributional concerns do not enter in the determination of optimal policy. This is due to the twin assumptions of a utilitarian social welfare function and Gorman-polar form preferences. Thus, any value for $\alpha \in[0,1]$ is as good as any other. The tax, on the other hand, does matter. Its optimal level is found by maximizing $W$ with respect to $q$. Routine algebraic manipulation leads to the second-best polluting-good tax characterized by,

$$
q^{o}-1=\frac{\varphi^{\prime}\left(\bar{y}\left(q^{o}\right)\right) \varepsilon / b\left(q^{o}\right)+\eta}{(1+\eta) \varepsilon-\eta} .
$$

where $\varepsilon$ is the absolute value of the price elasticity of aggregate demand, $\bar{y}(q)$, evaluated at $q^{o} .{ }^{10}$ Observe that if the capital and labor income taxes were non-distortionary, $\eta=0$, and the tax will be reduced to $q-1=\varphi^{\prime}(\bar{y}(q)) / b(q)$ which is the standard firstbest Pigouvian tax (tax equals the marginal social damage of the externality). In the second-best, the tax is adjusted by the marginal cost of public funds and the elasticity of demand for the polluting good. The tax would be positive as long as $\varepsilon>\eta /(1+\eta)$; a condition that holds empirically. ${ }^{11}$ The formula shows that, ceteris paribus, the tax moves negatively with the elasticity of demand for polluting good. Moreover, a higher marginal cost of public funds tends to call for a lower tax rate if the demand is relatively elastic, and a higher tax rate if the demand is inelastic. These are of course what one would intuitively expect.

\section{A voter's most-preferred $(q, \alpha)$}

We first consider $\theta^{\prime}$ 's most-preferred value of $\alpha$ conditional on $q, \alpha^{*}(\theta, q)$, and then his most-preferred $q$ conditional on $\alpha, q^{*}(\theta, \alpha)$. The voter's most-preferred $(q, \alpha)$ will then be the solution to $q=q^{*}\left(\theta, \alpha^{*}(\theta, q)\right)$ and $\alpha=\alpha^{*}\left(\theta, q^{*}(\theta, \alpha)\right)$.

\footnotetext{
${ }^{10}$ The second-order condition for this problem is

$$
b(q) \bar{y}^{\prime}\left[1+2 \eta-\frac{\eta \bar{y} \bar{y}^{\prime \prime}}{(1+\eta) \bar{y}^{\prime 2}}-\frac{\varphi^{\prime \prime}(\bar{y}) \bar{y}^{\prime}}{b(q)}+\frac{b^{\prime}(q) \varphi^{\prime}(\bar{y})}{b^{2}(q)}\right]<0,
$$

which we assume to be satisfied.

${ }^{11}$ The smallest reported estimate for $\varepsilon$ is 0.15 . Even with this small figure, the condition will be satisfied as long as $\eta<0.18$. See Fullerton (1991) who shows that-using the change in actual tax revenue and the equivalent variation measure for a welfare change in calculating the marginal cost of public funds - that $\eta$ varies between 0 to 0.12 .
} 


\subsection{Characterization of $\alpha^{*}(\theta, q)$.}

Lemma 1 characterizes $\alpha^{*}(\theta, q)$. It is obtained by differentiating (10) partially with respect to $\alpha$, making use of (9). Interestingly, it turns out, $q$ affects $\alpha^{*}(\theta, q)$ only through the sign of $R(q)$. This, in turn, depends on whether the polluting good is taxed $(q>1)$, or subsidized $(q<1)$. We have:

Lemma 1 An individual's most-preferred value of $\alpha$ is given by

$$
\begin{aligned}
& \alpha^{*}(\theta, q)=1 \text { if } \frac{w}{r}>\frac{\bar{w}}{\bar{r}} \text { and } R(q)>0, \\
& \alpha^{*}(\theta, q)=1 \text { if } \frac{w}{r}<\frac{\bar{w}}{\bar{r}} \text { and } R(q)<0, \\
& \alpha^{*}(\theta, q)=0 \text { if } \frac{w}{r}<\frac{\bar{w}}{\bar{r}} \text { and } R(q)>0, \\
& \alpha^{*}(\theta, q)=0 \text { if } \frac{w}{r}>\frac{\bar{w}}{\bar{r}} \text { and } R(q)<0, \\
& \alpha^{*}(\theta, q)=[0,1] \text { if } \frac{w}{r}=\frac{\bar{w}}{\bar{r}} \text { or } R(q)=0 .
\end{aligned}
$$

The intuition behind these expressions is straightforward. Recall from (10) that $\alpha$ affects $V(q, \alpha, \theta)$ directly through $\delta(\theta, \alpha)$ only. It is then plain that individual $\theta$ prefers $\delta(\theta, \alpha)$ to attain its maximum value if $R(q)>0$, and its minimum value if $R(q)<0$. Put differently, if the polluting good is taxed and the individual is to receive a refund, he would want the highest possible refund. On the other hand, if the polluting good is subsidized and the individual is to be taxed to finance it, he would want to pay the lowest possible (income) tax.

The first and fourth expressions in Lemma 1 postulate that $w / r>\bar{w} / \bar{r}$. Under this assumption, from $(9), \delta(\theta, \alpha)$ is highest when $\alpha=1$, and lowest when $\alpha=0$. That is, if the individual's share of labor income is larger than "average," he would want to link the refunds (if the voters are to get refunds) solely to labor incomes which would get him the highest refunds. And if he were to be taxed (on income), he would want to link the tax solely to capital incomes which would assure him the lowest tax payment.

In contrast, the assumption in the second and third expressions of Lemma 1 is $w / r<\bar{w} / \bar{r}$. Given this, $\delta(\theta, \alpha)$ is highest when $\alpha=0$, and lowest when $\alpha=1$. That is, if the individual's share of labor income is smaller than "average," he would want refunds to be based solely on capital income, and tax payments solely on labor incomes. 
Finally, if $w / r=\bar{w} / \bar{r}$, it will not matter on which income source taxes and refunds are based. In this case, the individual's utility is independent of $\alpha .{ }^{12}$

\subsection{Characterization of $q^{*}(\theta, \alpha)$.}

Lemma 2, proved in the Appendix, characterizes $q^{*}(\theta, \alpha)$.

Lemma 2 Let $\widetilde{q}(\delta, m)=\arg \max _{q}[a(q)+m b(q)+\delta b(q) R(q)-\varphi(\bar{y}(q))]$. We have $q^{*}(\theta, \alpha)=$ $\widetilde{q}(\delta(\theta, \alpha), m(\theta))$ so that $q^{*}$ depends on $\alpha$ only through $\delta$, with

$$
\begin{aligned}
\frac{\partial \widetilde{q}}{\partial \delta} & =\frac{b(q)\left[R^{\prime}(q)-d(q) R(q)\right]}{-\partial^{2} V(q, \alpha, \theta) / \partial q^{2}}, \\
\frac{\partial \widetilde{q}}{\partial m} & =\frac{b^{\prime}(q)}{-\partial^{2} V(q, \alpha, \theta) / \partial q^{2}} \leq 0 .
\end{aligned}
$$

It follows from Lemma 2 that if income effects on the demand for $y$ are negligible (as with quasi-linear preferences), richer individuals (regardless of the income source) prefer a higher tax. To see this, observe that with quasi-linear preferences, incomes affect $\widetilde{q}$ only through $\delta$. Moreover, assuming that rational agents do not want to be on the downward section of the "Laffer curve," $R^{\prime}(q)>0$ and $\partial \widetilde{q} / \partial \delta>0$. The result then follows from the fact that $\delta$ increases with $w$ and $r$. Now the presence of income effects imply that $\widetilde{q}$ moves negatively with $w$ and $r$ through (13). The net impact of income effects would then be to have taxpayers desiring a smaller tax rate or even a subsidy (as compared to the case with no income effects).

\section{The Political Competition Model: PUNEs}

The solution concept we use is John Roemer's "Party Unanimity Nash Equilibrium" (PUNE); see Roemer (2001). To make the paper self contained, we begin by giving a brief sketch of the main features of this equilibrium concept.

Two political parties compete for votes in an election. Each chooses a policy that it will implement if elected, and people vote for the party whose policy they prefer.

\footnotetext{
${ }^{12}$ If one plots individuals with different labor and capital incomes in a diagram with $w$ on the vertical axis and $r$ on the horizontal axis, then the line $w / r=\bar{w} / \bar{r}$ which represents the set of types for whom $\partial \delta(\theta, \alpha) / \partial \alpha=0$, divides the plane into two parts. People above this ray (northwest) would prefer $\alpha^{*}=1$ with a tax and $\alpha^{*}=0$ with a subsidy. On the other hand, people below this line (southeast) would prefer $\alpha^{*}=0$ with a tax and $\alpha^{*}=1$ with a subsidy.
} 
There are two departures from the classical Downs model. First, there is electoral uncertainty: when choosing their platform, the parties do not know for sure which party will, given the platforms, win the election. Each bases its platform selection, in part, on the probability of winning the election. Second, parties are not interested only in winning the election; they also care about the policies. More precisely, each party is composed of two factions: the "opportunists" and the "militants". These factions are not identified with particular types of voters. The opportunists aim to maximize the probability of winning the election; they are uninterested in policies per se. The militants, on the other hand, are only interested in the policies announced regardless of their electoral ramifications. They choose the policy that maximizes their "party's utility" (to be discussed below) without taking the electoral consequences into account.

Each faction has a complete preference order on the set of possible policies. The preference of the party is the intersection of these two orders. Thus, unanimity between the two factions is required for a party to accept a deviation from its current policy. This unanimity rule determines the preferences (payoffs) of the two parties who simultaneously choose their political platforms. A PUNE is a Nash equilibrium of this game.

To formally define a PUNE, index the parties by $i=L, R$. The objective function of the militants is defined as

$$
v_{i}(q, \alpha)=\int_{H} \omega_{i}(\theta) V(q, \alpha, \theta) f(\theta) d \theta, \quad i=L, R,
$$

where $\omega_{i}(\theta)$ is the weight attributed by party $i$ 's militants to individuals of type $\theta$. The probability that party $i$ wins the election is denoted by $\pi_{i}\left(q_{i}, \alpha_{i}, q_{j}, \alpha_{j}\right) ; i, j=L, R$, $i \neq j$. This probability increases with the share of voters preferring party $i$ 's policy to that of party $j$. It is determined by assuming that each individual $\theta$ participates in the election with some probability $\psi(\theta)$. Further, assume that when both parties offer the same policy, $\pi_{L}=\pi_{R}=1 / 2$. The probability of winning the election represents the objective function of the opportunists. We have, ${ }^{13}$

\footnotetext{
${ }^{13}$ Roemer (2001) considers a third faction, namely the "reformists," who care for the party's expected utility. They choose the electoral platform that maximizes the party's utility, taking into account the probability of winning the election with this platform. The objective of party $i$ 's reformists is thus given
} 
Definition 1 Let $T=\mathbb{R}^{+} \times[0,1]$ be the policy space. A Party Unanimity Nash Equilibrium is a pair of admissible policies $\left(q_{L}, \alpha_{L}\right),\left(q_{R}, \alpha_{R}\right) \in T$ such that for each $i, j=L, R, i \neq j ; \nexists(q, \alpha) \in T$ with the property that, given $\left(q_{j}, \alpha_{j}\right), v_{i}(q, \alpha) \geq v_{i}\left(q_{i}, \alpha_{i}\right)$ and $\pi_{i}\left(q, \alpha, q_{j}, \alpha_{j}\right) \geq \pi_{i}\left(q_{i}, \alpha_{i}, q_{j}, \alpha_{j}\right)$, where there is at least one strict inequality.

Following Roemer (2001), we shall restrict our attention to regular PUNEs which are defined as,

Definition 2 A regular PUNE is a pair of admissible policies $\left(q_{L}, \alpha_{L}\right),\left(q_{R}, \alpha_{R}\right) \in T=$ $\mathbb{R} \times[0,1]$ that are PUNEs, and additionally satisfy the following conditions:

(i) for each $i, j=L, R ; v_{i}\left(q_{i}, \alpha_{i}\right) \geq v_{i}\left(q_{j}, \alpha_{j}\right)$;

(ii) for each $i, j=L, R ; 0<\pi_{i}\left(q_{i}, \alpha_{i}, q_{j}, \alpha_{j}\right)<1$.

Regularity is thus imposed as an additional requirement which refines the equilibrium concept and (potentially) reduces the set of equilibria. The first condition states that the militants of each party prefer the policy of that party to that of the other party (militants of, say, $L$ prefer $\left(q_{L}, \alpha_{L}\right)$ to $\left.\left(q_{R}, \alpha_{R}\right)\right)$. This requirement, while quite sensible, is not automatically satisfied by all PUNEs. The reason is that a switch to the other party's platform could decrease the probability of winning and thus be vetoed by the opportunists. The second condition is essentially a technical requirement which is meant to eliminate some pathological equilibria.

\section{Data and calibrations}

In order to compute the PUNEs, we must know the voters' incomes and preferences. Additionally, we should know the parties' militants' preferences and the probability that a voter of a particular type would participate in the election. We take our voters to be US households as represented by the 2001 Panel Study for Income Dynamics Survey.

by

$$
\pi_{i}\left(q_{i}, \alpha_{i}, q_{j}, \alpha_{j}\right) v_{i}\left(q_{i}, \alpha_{i}\right)+\left(1-\pi_{i}\left(q_{i}, \alpha_{i}, q_{j}, \alpha_{j}\right)\right) v_{i}\left(q_{j}, \alpha_{j}\right) \quad i, j=L, R, i \neq j .
$$

This expression shows that the reformists are purely gratuitous in this model: If both opportunists and militants agree to a deviation, reformists will do so as well. This occurs because when both $\pi_{i}$ and $v_{i}\left(q_{i}, \alpha_{i}\right)$ increase, expected utility also increases.

Since the presence (or the absence) of reformists does not affect the results, we have opted for not introducing them. 
The survey consists of 7,406 households each of whom is assigned a weight to make the sample representative of the US population in 2000. Naturally, we consider only those who reported a nonzero income for the year 2000 (whether labor or asset incomes). ${ }^{14}$ These total 6,877 households. We then fit a bivariate lognormal distribution for labor and asset incomes to this truncated sample (while using the same weights that the survey assigns to each household. $)^{15}$

The next task is to calculate numerical values for the parameters of the individuals' utility function. The polluting good is called energy and consists of an aggregate of energy-related consumption goods (fuel oil, gasoline, natural gas, kerosene, LPG and electricity). Given the Gorman-polar specification, we have $y(q)=-a^{\prime}(q) / b(q)-$ $b^{\prime}(q) / b(q) I$. Assume $b(q)=1-\beta q$. This allows us to capture the difference between Gorman-polar and quasi-linear specifications for preferences through a single parameter, $\beta$. The demand function is thus written as

$$
y(q)=\frac{-a^{\prime}(q)}{1-\beta q}+\frac{\beta}{1-\beta q} I .
$$

Assuming that the first expression in the right-hand side of above is linear in price (with a constant term), we calculate the three parameters of the resulting equation (constant term, coefficient of $q$, and the parameter $\beta$ ) on the basis of a marginal propensity to consume energy out of income equal to $2.25 \%$ (equal to a value of 0.405 for the income elasticity of demand for the "average consumer"), price elasticity of demand equal to -0.30 , and a ratio of average expenditure on energy to average income equal to 0.0555 , with the average income being $\$ 59,926 .{ }^{16}$ All our calculations are based on the

\footnotetext{
${ }^{14}$ Specifically, we calculate labor incomes as the sum of labor income, and labor part of the business income, of the Head of the household and his spouse. To calculate the households' asset incomes, we subtract each family's labor income (as we have calculated it), transfer income, social security income and the Head's farm income, from the family's reported total income.

${ }^{15}$ Recall that our model postulates that voters differ only in two dimensions: labor and asset incomes. The mean, median and standard deviation are $\$ 50,294, \$ 36,100$ and $\$ 64,825$ for labor incomes, and $\$ 9,632, \$ 433$, and $\$ 42,838$ for asset incomes. The Correlation coefficient between labor and asset incomes is 0.163 - a figure which is in line with the numerical calculation of Champernowne and Cowell (1998) who report a correlation coefficient of 0.135 using 1985 PSID data.

${ }^{16}$ The -0.30 figure is based on the literature estimates for the price elasticity of consumer demand for energy. These vary from -0.35 to -0.15 ; see Branch (1993), Filippini (1999), Gately and Huntington (2001), Hodge (1999), National Institute of Economics and Industry Research (2002), Ninomiya (2002). The 0.0555 figure is found from the Consumer Expenditure Surveys, 2002, which report a value of 0.064 for the ratio of average energy consumption to average annual expenditures; and 0.8667 for the ratio of
} 
assumption that the relative consumer price of a "unit" of energy is equal to one. This normalization implies that all the predicted tax rates are in addition to current taxes.

Considering the disutility from pollution, we assume that it is increasing and convex in $Y$, with the specification

$$
\varphi(Y)=e^{h+k Y} .
$$

We take the pollution generated by energy to be the release of carbon dioxide into the atmosphere. The carbon content of the polluting good is found according to the carbon content of each appropriately-weighted component. Using a value of $\$ 50$ for the social marginal cost of a ton of carbon, ${ }^{17}$ we are able to calculate the marginal social damage of one unit of the polluting good. This translates into a first-best tax rate of about $10 \%$ on the polluting good This leaves one degree of freedom in setting $h$ and $k$. We use it by choosing the least convex function compatible with a positive value for every household's most-preferred $q$.

Turning to the value of the marginal cost of public funds in the US, we use a value of 0.025 for $\eta$. This is within the range of the existing estimates in the literature, though rather on the low side; see, e.g., Fullerton (1991) who reports a figure between 0 and 0.12 . However, recall that in our formulation $\eta(q-1) \bar{y}(q)$ measures the revenue gained by raising $(q-1) \bar{y}(q)$ from emission taxes while reducing income taxes by the same amount. Now, the double-dividend debate is inconclusive on whether this tax recycling does not in fact exacerbate the existing distortions in the economy rather than mitigating it. To the extent that the general equilibrium effects of this tax switch (which we do not allow for) lower the potential gains, one should use a low value for $\eta$. Nevertheless, while we set $\eta=0.025$ in our benchmark case, we also set $\eta$ equal to zero and 0.20 (which far exceeds the maximum estimate reported by Fullerton) for our sensitivity analyses. Observe also that, with $\eta=0.025$, the second-best emission tax is about $16 \%$.

Next, we turn our attention to the militants' preferences. Given the definition of $v_{i}(\tau)$ in (14), we need to determine the weights that the two parties' militants assign to the preferences of each voter, $\omega_{i}(\theta)$. These we find from Bartels (2002). He estimates a average net-of-tax to average gross-of-tax income. The $\$ 59,926$ value for average income comes from the 2001 PSID data.

${ }^{17}$ This is within the range of estimated values of $\$ 5.5$ to $\$ 187$; see http://www.epa.gov/oppt/epp/guidance/top20faqexterchart.htm on the EPA website. 
linear relationship between the ideology (measured on a single dimension) of a senator and the ideology of his constituents, with different constituents being assigned different weights based on their incomes. He runs this regression separately for democratic $(L)$ and republican $(R)$ senators for three consecutive sessions of the Congress, 101-103. The weights (a weighted average of the weights derived for the three sessions of the Congress) are

$$
\begin{aligned}
& \omega_{L}=-0.02+0.04 * \text { income } \\
& \omega_{R}=-0.86+0.099 * \text { income. }
\end{aligned}
$$

To apply these to our setting, we modify the weights in two directions. First, the coefficients of incomes are deflated by the variation in the Consumer Price Index between 1990 and 2000. That is, we divide 0.04 and 0.099 above by 1.278 . This is to correct for the fact that Bartels uses incomes expressed in 1990 dollars to calculate the weights, but in our calculations we use PSID data for the year 2000. Second, we restrict $\omega_{i}$ to be nonnegative. Thus, for the Democrats, we set the constant part of $\omega_{L}$ at zero (instead of -0.02); and for the Republicans, we use $\max \left(0, \omega_{R}\right)$ rather than $\omega_{R} \cdot{ }^{18}$

Finally, to calculate the probability that a particular voter participates in the election, we continue to rely on Bartels (2002) using his regression of turnout on income. As with the voters' weights, we modify his results (as reported in his Table A6) on the basis of the consumer price indices for 1990 and 2000. Moreover, given the linear specification between turnout and income, there is also a need to cap the very rich individuals' estimated probability of turn out at one. The average turnout in the economy is then $72 \%$, and the "average individual" (a person with average income regardless of the source) participates in the election with a probability of $75.5 \%$.

\section{Results}

With each party proposing a two-dimensional policy, PUNEs can differ along four dimensions: $q_{L}, \alpha_{L} ; q_{R}, \alpha_{R}$. As such, they may be classified in numerous different ways.

\footnotetext{
${ }^{18}$ Bartels calculates these weights based on individuals' total incomes, not differentiating between labor and capital incomes. In using them, we thus assume that the weights the two parties assign to an individual are conditioned on his total income $w+r$ independently of the source of the income.
} 
As far as $q_{i}(i=L, R)$ is concerned, the most informative distinction is between taxes $\left(q_{i}>1\right)$ and subsidies $\left(q_{i}<1\right)$. Specifically, along this dimension, we distinguish between three potential types of results: Both parties propose a tax, both parties propose a subsidy, one party (either Left or Right) proposes a tax and the other party a subsidy.

Regarding $\alpha_{i}$, a useful distinction is between corner solutions of $\alpha_{i}=1$ or $\alpha_{i}=0$, the voters' most-preferred values for $\alpha_{i}$, and interior solutions where $0<\alpha_{i}<1$. An interior solution arises when one or both parties decide to offer a "compromise" in order to placate the militants in the party by pushing them closer to their blisspoint(s), and/or the opportunists in an attempt to win more votes in the election. As will be seen below, a large subset of regular PUNEs have this feature. We thus categorize the potential solutions into three types along this dimension as well: Both parties propose a corner solution for $\alpha_{i}$; one party (either Left or Right) proposes a corner value and the other an interior value for $\alpha_{i}$; both the Left and the Right parties propose an interior value for $\alpha_{i}$.

With three configurations each for $\left(q_{L}, q_{R}\right)$ and $\left(\alpha_{L}, \alpha_{R}\right)$, there will be, potentially, nine different solution categories. To calculate the PUNEs, we draw at random a huge number (in the thousands) of possible vectors $\left(q_{L}, \alpha_{L} ; q_{R}, \alpha_{R}\right)$ within each of the nine possible categories, and check whether a particular draw constitutes a PUNE. ${ }^{19}$ It turns out that (regular) PUNEs are of two types. In one type (Type I), the parties offer a tax in conjunction with $\alpha_{L}=\alpha_{R}=0$. In the other (Type II), both parties offer a subsidy coupled with an interior value for $\alpha$. See Figure 1. Most importantly, these features are very robust and remain intact for the various simulations we perform.

\subsection{Type I regular PUNEs}

Amongst the double corner proposals, only $\alpha_{L}=\alpha_{R}=0$ leads to regular PUNEs: There are no PUNEs when $\alpha_{L}$ and $\alpha_{R}$ are both equal to one; nor is it possible to have an equilibrium with one of the $\alpha_{i}$ 's equal to zero and the other equal to one. Moreover,

\footnotetext{
${ }^{19}$ Following Roemer(2001), we compute the PUNEs through a local characterization that makes use of Farkas' lemma. This local characterization ensures that no party has an incentive to deviate locally, and nor globally if the program solved were globally convex. It is well known, however, that the probability of winning functions, $\pi_{i}$ 's, are not in general quasi-concave. We will thus contend ourselves with identifying local PUNEs.
} 


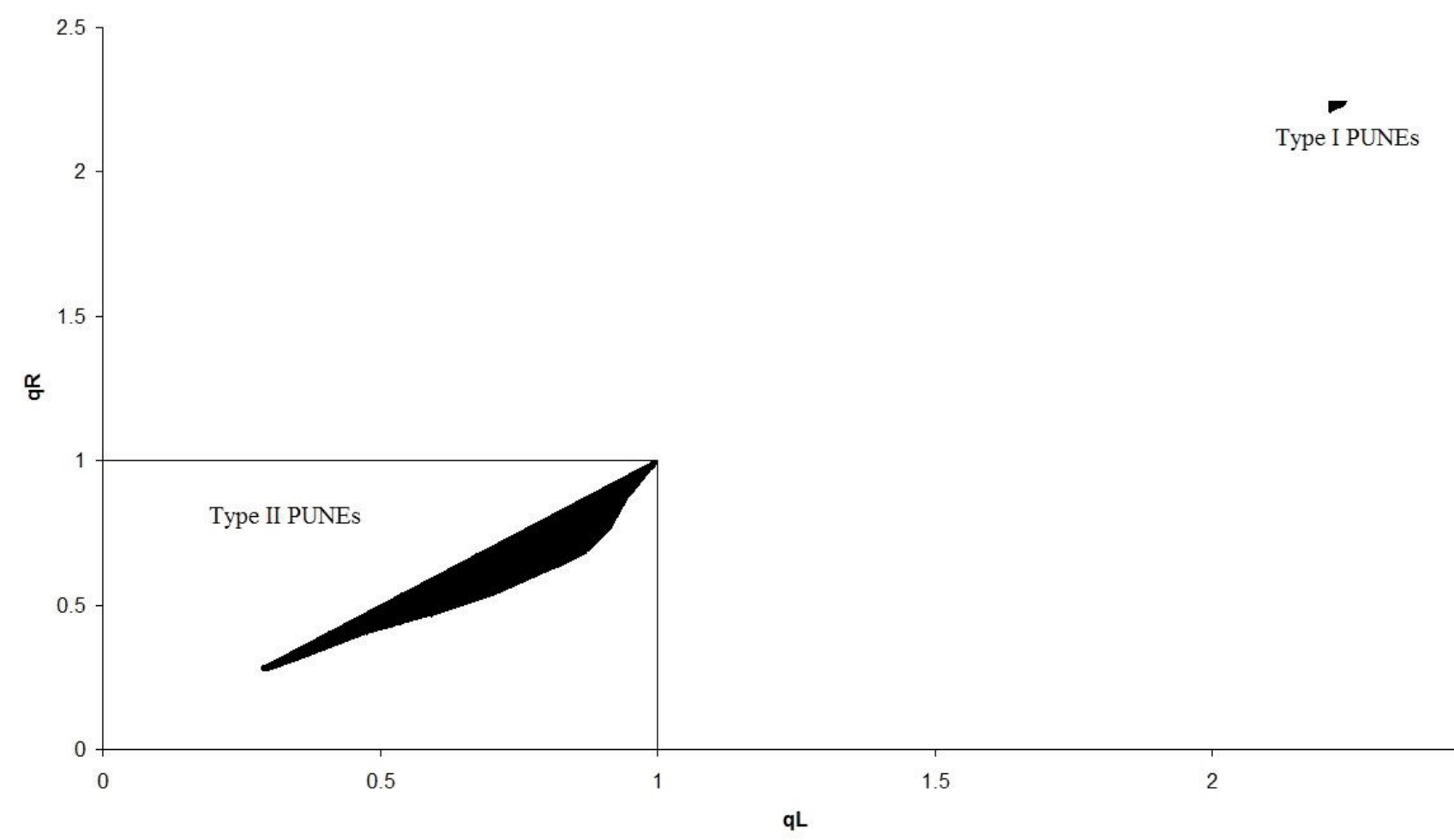

Figure 1: Type I and Type II PUNEs

along with $\alpha_{i}=0$, both parties will offer a tax; no equilibrium exists when one or both parties offer a subsidy. Specifically, all PUNEs with both $\alpha_{L}$ and $\alpha_{R}$ taking corner solutions consist of four-tuples $\left(q_{L}, 0, q_{R}, 0\right)$ such that $q_{L}$ and $q_{R}$ lie in the closed interval between each party's militants' most-preferred $q$ conditional on $\alpha=0$. More precisely, we have $2.19 \leq q_{L}<q_{R} \leq 2.22$, where the most-preferred value of $q$ conditional on $\alpha=0$ is $q_{L}^{*}=2.19$ for the Left party, and $q_{R}^{*}=2.22$ for the Right party. Additionally, $q_{L}=q_{R}=2.19$ is also a PUNE. We shall refer to these solutions as Type I (regular) PUNEs.

The properties of Type I PUNEs follow from four important properties of our calibrated model. First, income effects are "small" enough so that $q^{*}(\theta, \alpha)=\widetilde{q}(\delta(\theta, \alpha), m(\theta))$ is increasing in $w$ and $r$ for $q_{L}^{*} \leq q_{L} \leq q_{R} \leq q_{R}^{*}$. Second, preferences are single-peaked 
in $q$ (for a given $\alpha$ ). Third, for a given $q_{R}$, an increase in $q_{L}$ increases the proportion of the electorate who prefer $q_{L}$ to $q_{R}$; while, for a given $q_{L}$, an increase in $q_{R}$ lowers the proportion of the electorate who prefer $q_{R}$ to $q_{L}$. Fourth, given that the weights used by both parties' militants are increasing in total income, and that the Right party's militants put a zero weight on the utility of the poorer individuals, it follows that $q_{L}^{*}<q_{R}^{*}$. Observe that the second property also implies that a (classical Downsian) majorityvoting equilibrium exists, and that the utility of militants in party $i=L, R$ increases when their party's proposed $q$ moves closer to their blisspoint $q_{i}^{*}$.

For the purpose of comparison, we also calculate the majority-voting equilibrium value of $q$ conditional on $\alpha=0$, denoted by $q^{M}$. This is equal to $q^{M}=0.089$. Not surprisingly, with the median wage and the median capital income being respectively smaller than the average wage and the average capital income, $q^{M}<q^{o}$. Observe that the determination of $q^{M}$ is based on the assumption that all citizens participate in the election with the same probability. As we noted in Section 6, however, this is not the case empirically. Instead, turnout probability increases with total income. This suggests that more than $50 \%$ of voters (as opposed to citizens) prefer $q$ to be higher than $q^{M}$. We can then determine a second value for the majority-voting equilibrium $q$ which takes the turnout probabilities into account. Denoting this equilibrium by $q^{M V}$, we calculate $q^{M V}=0.095$. Observe that $q^{M}<q^{M V}<q^{o}$. This occurs because adjusting for turnout probabilities leaves the ratio of median income to average income, for both wage earners and capital owners, well below one. ${ }^{20}$

Given the above properties, it is easy to see why any $\left(q_{L}, q_{R}\right)$ in the interval $q_{L}^{*} \leq$ $q_{L}<q_{R} \leq q_{R}^{*}$ is a PUNE. To begin with, the most-preferred policies of the two parties' militants $\left(q_{L}^{*}, q_{R}^{*}\right)$ constitute, by definition, a PUNE: All deviations from this pair of policy proposals would decrease the utility of the militants in both parties. Secondly, $q_{L}^{*}=q_{L}=q_{R}$ is also a PUNE: Party L's militants oppose any deviation from this point, while R's opportunists block their party's militants' wish to increase $q_{R}$. Now take any $\left(q_{L}, q_{R}\right)$ with $q_{L}^{*}<q_{L}$ and consider how the factions in party $\mathrm{L}$ react to a

\footnotetext{
${ }^{20}$ Specifically, the ratio of median income to average income increases from $61 \%$ (for median citizen) to $73 \%$ (for median voter) in case of wage incomes, and from $22 \%$ (median citizen) to $26 \%$ (median voter) for capital incomes.
} 
decrease in $q_{L}$. This would be supported by L's militants as it brings them closer to their blisspoint; on the other hand, the opportunists in party L would oppose this move as it decreases the party's probability of winning. Similarly, take any $\left(q_{L}, q_{R}\right)$ with $q_{R}<q_{R}^{*}$ and consider party R's factions: Militants would like to increase $q_{R}$ while opportunists would prefer to decrease it. Allowing $\alpha_{i}$ to change as well, does not create deviations that simultaneously increase a party's probability of winning and its militants' utility. Intuitively, this occurs because $\alpha_{i}$ is on the boundary of the feasible set ( $\alpha_{i}$ does not take negative values) which limits the set of admissible deviations.

Other $q_{i}$-configurations besides those stated above are, however, not PUNEs. It is easy, for example, to see that no configuration with $q_{L}^{*}<q_{L}=q_{R}$ can be a PUNE. Starting from such a configuration, party L can increase both the utility of the militants and the party's probability of winning by decreasing $q_{L}$. As a second example, consider $q_{L} \leq q_{L}^{*}<q_{R} \leq q_{R}^{*}$. In this case, both the militants and the opportunists in the Left party want to increase $q_{L}$. Similar arguments rule out all other configurations with $\alpha_{L}=\alpha_{R}=0$. Observe also that despite the limitations imposed by corner values on the set of admissible deviations, other corner configurations beside $\alpha_{L}=\alpha_{R}=0$ contain no PUNEs. When one or both $\alpha_{i}$ 's $(i=L, R)$ are equal to one, the ability to reduce $\alpha_{i}=1$ will be sufficient to create possibilities for simultaneously increasing a party's probability of winning and its militants' utility so that no such configuration can constitute a PUNE.

Type 1 regular PUNEs are essentially dictated by the preferences of the parties' militants which, as observed earlier, are very far from the majority-voting equilibria $q^{M}$ and $q^{M V}$. Summing up, we have:

Result 1 Let $q_{L}^{*}$ and $q_{R}^{*}$ denote the Left and the Right parties' militants' most-preferred prices conditional on $\alpha=0$. Type I (regular) PUNEs are characterized by $\alpha=0$, and $2.21=q_{L}^{*} \leq q_{L}<q_{R} \leq q_{R}^{*}=2.23$, plus $q_{L}=q_{R}=q_{L}^{*}=2.21$.

Observe that with $q^{M}<q^{o}<q_{L}<q_{R}$, the majority of the electorate will vote for the Left party. Specifically, the Left party has a probability of winning the election that varies from $90.8 \%$ to $91.2 \%$ across all possible configurations of $q_{L}$ and $q_{R}$, with an average value of $91 \%$. 


\subsection{Type II regular PUNEs}

When both $\alpha_{L}$ and $\alpha_{R}$ take interior values, a second type of PUNEs emerges involving both parties offering a subsidy. It will not be possible to have one or both parties offering a tax in combination with interior values for $\alpha_{L}$ and $\alpha_{R}$. Specifically, all PUNEs of Type II share the following characteristics: $0<q_{R}<q_{L}<1$ and $0<\alpha_{L}<\alpha_{R}<1$. Unlike Type I PUNEs, it is the opportunists in the two parties who play the major role in determining these PUNEs. With $q_{L}$ varying between 0.291 and 0.995 and $q_{R}$ between 0.284 and 0.992 , these PUNEs are closer to the most-preferred policy of an individual with the median wage and the median capital income. To see who votes for which party, recall that this choice is essentially determined by two factors. First, the lower subsidy offered by party L attracts the higher income voters (aggregate income effect). Second, individuals who derive a higher proportion of their income from wages favor party L's proposed budgetary rule while those with high capital incomes prefer party R's (income composition effect). This is the case because with both parties offering a subsidy on the polluting good, whether the Left or the Right party wins, the voters know that they will have to be taxed in order to finance the environmental subsidy. With a high $\alpha$ punishing the high-wage-earners more and a low $\alpha$ punishing the high-capital-income individuals more, the former group will want a low $\alpha\left(\alpha_{L}\right)$ and the latter a high $\alpha\left(\alpha_{R}\right)$.

For certain voters, both factors reinforce each other. Those with a high wage income and low capital income prefer party L's policy to party R's, while individuals with low total income but a high share of capital income prefer party R's policy. For other voters, the two effects go in opposite directions. In all these cases, however, the income composition effect dominates the aggregate income effect. The set of voters who are indifferent between the two parties' policies is thus given by an upward-sloping line in the $(w, r)$ space. All individuals above this line (i.e., relatively more endowed in capital income) prefer party R's policy while individuals below it prefer party L's. Put differently, with Type II PUNEs, it is the electorate's composition of wage and capital incomes that polarizes the society.

The end outcome in terms of attracting voters appears to be very close for the two parties, with a small edge to party $L$. It faces a probability of winning that ranges from 
$48.9 \%$ to $69.6 \%$ (depending on the particular regular PUNE that is selected). Moreover, assuming each PUNE is equiprobable, party $L$ 's average probability of winning (among all PUNEs) is $54.1 \%$. Naturally, party $R$ 's probability of winning varies from $30.4 \%$ to $51.1 \%$ with an average rate of $45.9 \%$. These probabilities are, intuitively, very comforting. At $54.1 \%$ and $45.9 \%$, the average probabilities of winning for the two parties are rather close. Moreover, the maximum winning probabilities for the two parties over the set of Type II PUNEs exceed, while their minimum winning probabilities fall below, the $50 \%$ mark.

Summing up, we have obtained the following main results

Result 2 Type II PUNEs share the following characteristics: $0<\alpha_{L}<\alpha_{R}<1$ (both parties offer an interior solution) and $q_{R}<q_{L}<1$ (both parties offer a subsidy); specifically, $q_{L}$ varies from 0.295 to 0.995 and $q_{R}$ from 0.284 to 0.992. Party L's average probability of winning (among all PUNEs) is 54.1\%, and party $R$ 's is $45.9 \%$.

Finally, three interesting features of these PUNEs are worth emphasizing. One is the fact that they all entail a subsidy on the environmental good. The subsidies range from $0.5 \%$ to $70.9 \%$ for $q_{L}$ (with an average of $24.9 \%$ ) and from $0.8 \%$ to $71.6 \%$ for $q_{R}$ (with an average of $30.9 \%$ ). These figures underscore the importance of distributional concerns in ensuring political support for environmental policies. The literature on environmental taxation, with few exceptions, has shied away from such considerations. ${ }^{21}$ In our setup, on the other hand, distributional issues take the center stage.

A second interesting feature of Type II PUNEs is that the two parties always compromise in their choices of $\alpha$, offering an interior solution. Specifically, $\alpha_{L}$ varies from 0.01 to 0.98 with an average of 0.425 while $\alpha_{R}$ varies from 0.1 to 0.995 with an average of 0.69 . This aspect too underlines the importance of generating political support for environmental policies (through political compromise). The compromise over $\alpha$ turns out to be a particularly striking feature of the PUNEs; most other equilibrium concepts, even when they are not empty in multidimensional choice sets, do not share this feature;

\footnotetext{
${ }^{21}$ In particular, the burgeoning literature of the past decade has emphasized efficiency issues that arise in second-best settings; see the many papers that appear in the edited volume by Goulder (2003). Cremer, Gahvari and Ladoux (2003) is one exception.
} 
see Cremer et al. (2004b). ${ }^{22}$

Third, and most interestingly, we observe that Type II PUNEs correspond to the observed making of environmental tax policy in the US. That is, the policy has been dictated more by the concerns of the opportunists who care most about winning elections. However, the existence of Type I PUNEs suggests the possibility of a change in this policy and arriving at an equilibrium with the militants at the helm.

\subsection{Income effects and distortions}

To investigate the robustness of our results, we have calculated, in addition to our benchmark case, the set of PUNEs for two extreme values of the income elasticity of demand for the average consumer, $\zeta$, and two extreme values of $\eta$. These are reported in Tables 1 and 2. Starting with the reported numbers in Table 1, the striking feature of our results is their robustness to income effects. The same two sets of PUNEs, Type I and Type II, comprise the political equilibria. Type I PUNEs are characterized by a huge tax with $\alpha_{L}=\alpha_{R}=0$, and Type II by a subsidy with interior solutions for $\alpha_{L}, \alpha_{R}$. Observe also that in the case of Type I PUNEs, $q_{L}^{*}$ and $q_{R}^{*}$ both decline as $\zeta$ increases. This follows our theoretical result in Lemma 1 concerning the direct negative relationship between income and the most-preferred values of $q$. The figures also show that as $\zeta$ increases, the probability of winning of the Left party decreases under Type I PUNEs and increases under Type II PUNEs.

Turning next to the effects of $\eta$, we again note that our results are remarkably robust; The same two PUNE types emerge with the exact same characteristics. Observe that in this case as $\eta$ increases, $q_{L}^{*}$ and $q_{R}^{*}$ also increase. This is in contrast with the effect of an increase in $\zeta$. The reason is that an increase in $\eta$ effectively lowers real incomes and thus has the same effect as a decrease in $\zeta$. Nevertheless, we observe that, as with $\zeta$, increasing $\eta$ causes the probability of winning of the Left party to decrease under Type I PUNEs and to increase under Type II PUNEs.

\footnotetext{
${ }^{22}$ This is also the case for probabilistic voting which results in $\alpha=0$; see Cremer et al. (2005). Levy (2004) introduces a model wherein candidates are citizens who can only commit to implement their most-preferred policy, and parties are coalitions of candidates restricted to propose policies inside their Pareto set. In this setup, one could obtain an interior $\alpha$ as an equilibrium provided that it belongs to the Pareto set of some coalition of citizens. Applying this framework in our model with a continuum of citizens will be a rather impossible task.
} 
Table 1. Income effects and the PUNEs:

$$
(\eta=0.025)
$$

\begin{tabular}{|c|c|c|c|c|c|c|c|c|}
\hline \multicolumn{3}{|c|}{$\begin{array}{c}\text { Type 1 } \\
\left(\alpha_{L}=\alpha_{R}=0\right)\end{array}$} & \multicolumn{6}{|c|}{ Type 2} \\
\hline \multicolumn{9}{|c|}{$\zeta=0$} \\
\hline$q_{L}^{*}$ & $q_{R}^{*}$ & $\pi_{L}$ (average) & & $\alpha_{L}$ & $q_{L}$ & $\alpha_{R}$ & $q_{R}$ & $\pi_{L}$ (average) \\
\hline \multirow[t]{2}{*}{2.4851} & 2.5141 & 0.9551 & Min: & 0.0033 & 0.3029 & 0.0607 & 0.2884 & 0.5181 \\
\hline & & & Max: & 0.8588 & 0.9929 & 0.9969 & 0.9876 & \\
\hline \multicolumn{9}{|c|}{$\zeta=0.405$} \\
\hline \multirow[t]{2}{*}{2.2119} & 2.2345 & 0.9099 & Min: & 0.0047 & 0.2910 & 0.1005 & 0.2842 & 0.5405 \\
\hline & & & Max: & 0.9873 & 0.9949 & 0.9988 & 0.9924 & \\
\hline \multicolumn{9}{|c|}{$\zeta=0.810$} \\
\hline \multirow[t]{2}{*}{1.9448} & 1.9615 & 0.7094 & Min: & 0.0061 & 0.5453 & 0.1064 & 0.5110 & 0.6111 \\
\hline & & & Max: & 0.9321 & 0.9931 & 0.9962 & 0.9848 & \\
\hline
\end{tabular}

\subsection{Other equilibria?}

In subsection 7.1 we argued that Type I PUNEs are the only equilibria of our model when $\alpha_{L}$ and $\alpha_{R}$ both take corner values. Here we shall discuss why Type II PUNEs will be the only possible equilibria when $\alpha_{L}$ and $\alpha_{R}$ both take interior values. We will also discuss why no equilibria exists when either $\alpha_{L}$ or $\alpha_{R}$ takes a corner value, and the other an interior value. To develop an intuition for these results, we first study the reasons as to why a Type II PUNE constitutes an equilibrium so that neither party wants to deviate from such an allocation.

\subsubsection{Type II PUNEs as equilibria}

At an interior PUNE, we have the gradient of the utility of a party's militants, in $(q, \alpha)$ space, to be exactly opposite to the gradient of that party's probability of winning. Considering each party's militants' most profitable deviations, we note that their mostpreferred values of $q_{i}$ are larger than one (they prefer a tax) whatever the value of $\alpha_{i}$. Now, given that Type II PUNEs are characterized by both parties offering a $q_{i}<1$, the militants in both parties would always prefer a larger $q_{i}$. As for $\alpha_{i}$, the militants most prefer $\alpha_{i}=1$ when $q_{i}<1$. Consequently, they will also want to increase $\alpha_{i}$.

Turning next to the opportunists, what they would wish to do depends on the $(w, r)$ 
Table 2. Distortionary income taxes and the PUNEs:

$(\zeta=0.405)$

\begin{tabular}{|c|c|c|c|c|c|c|c|c|}
\hline \multicolumn{3}{|c|}{$\begin{array}{c}\text { Type 1 } \\
\left(\alpha_{L}=\alpha_{R}=0\right)\end{array}$} & \multicolumn{6}{|c|}{ Type 2} \\
\hline \multicolumn{9}{|c|}{$\eta=0$} \\
\hline$q_{L}^{*}$ & $q_{R}^{*}$ & $\pi_{L}$ (average) & & $\alpha_{L}$ & $q_{L}$ & $\alpha_{R}$ & $q_{R}$ & $\pi_{L}$ (average) \\
\hline \multirow[t]{2}{*}{2.1977} & 2.2212 & 0.9104 & Min: & 0.0004 & 0.3360 & 0.0750 & $\overline{0.3210}$ & 0.5220 \\
\hline & & & Max: & 0.9158 & 0.9920 & 0.9980 & 0.9860 & \\
\hline \multicolumn{9}{|c|}{$\eta=0.025$} \\
\hline \multirow[t]{2}{*}{2.2119} & 2.2345 & 0.9099 & Min: & 0.0047 & 0.2910 & 0.1005 & 0.2842 & 0.5405 \\
\hline & & & Max: & 0.9873 & 0.9949 & 0.9988 & 0.9924 & \\
\hline \multicolumn{9}{|c|}{$\eta=0.2$} \\
\hline \multirow[t]{2}{*}{2.2943} & 2.3122 & 0.9068 & Min: & 0.0010 & 0.3711 & 0.1391 & 0.3590 & 0.5999 \\
\hline & & & Max: & 0.9891 & 0.9708 & 0.9988 & 0.9592 & \\
\hline
\end{tabular}

distribution among the voters. This distribution determines, for a given pair of policy proposals, how many people vote for each party. It turns out that the $(w, r)$ distribution is such that a party's choice of $\alpha$ will be the more crucial parameter in securing votes for the party's platform. Now given that $q_{i}<1$ for both parties, an increase in $\alpha_{i}$ by any party appeals more to people who have a lot of capital income. That capital income is positively skewed then implies that by increasing its proposed value of $\alpha_{i}$, a party loses more votes (among the low- $r$ people) than it gains (among large- $r$ people), thus lowering its probability of winning the election. This result holds true for all PUNEs even though the turnout probability increases with income. As a consequence, opportunists in both parties would like to decrease $\alpha_{i}$ in order to attract the votes of the more numerous low$r$ people. The same reasoning applies to an increase in $q_{i}$ by any party. High-income people benefit, and low-income people lose, from an increase in $q$. With incomes being positively skewed, the party that increases $q_{i}$ loses more votes among poor people than it gains among richer people. Consequently, opportunists in both parties would prefer to decrease their $q_{i}$ as well in an attempt to increase their probability of winning.

\subsubsection{Opposing tax/subsidy proposals accompanying interior values of $\alpha$}

Interestingly, there are no regular PUNEs with this property. That is, there are no fourtuples $\left(q_{L}, \alpha_{L}, q_{R}, \alpha_{R}\right)$ with $q_{L}<1<q_{R}$ such that party $L$ 's militants prefer $\left(q_{L}, \alpha_{L}\right)$ 
to $\left(q_{R}, \alpha_{R}\right)$. Nor are there any four-tuples $\left(q_{L}, \alpha_{L}, q_{R}, \alpha_{R}\right)$ with $q_{R}<1<q_{L}$ such that party $R$ 's militants prefer $\left(q_{R}, \alpha_{R}\right)$ to $\left(q_{L}, \alpha_{L}\right)$. Intuitively, because both $L$ 's and $R$ 's militants put more weight on higher income people, they both prefer a tax to a subsidy whatever the proposed value of $\alpha$.

\subsubsection{Tax proposals by both parties accompanying interior values of $\alpha$}

The militants of a party prefer to combine a tax with a lower value of $\alpha$ (see the proof of Result 1). Additionally, in all regular 4-tuples $\left(q_{L}, \alpha_{L}, q_{R}, \alpha_{R}\right)$ party $L$ 's militants always prefer a higher value of $q_{L}$ regardless of the value of $\alpha$. Party R's militants, on the other hand, may prefer a lower as well as a higher value of $q_{R}$ (i.e., $q_{R}$ may exceed party $R$ 's militants' most-preferred value of $q$ in regular 4 -tuples). Turning to the opportunists, their behavior varies according to the circumstances they face: there exist regular four-tuples for which party $i$ 's $(i=L, R)$ probability of winning increases with both $\alpha_{i}$ and $q_{i}$, regular four-tuples for which this probability decreases with both $\alpha_{i}$ and $q_{i}$, and yet other regular four-tuples for which this probability increases with $q_{i}$ and decreases with $\alpha_{i}$, or the opposite. Given these properties, there exist no PUNE with both parties proposing a tax: there is no four-tuple of this type for which party $L$ 's opportunists want to block the militants by wanting to increase $\alpha_{L}$ and decrease $q_{L}$, while at the same time party $R$ 's opportunists want to go in a direction opposite to that favored by $R$ 's militants. In other words, when both parties offer interior values for $\alpha$ coupled with taxes, one can always find deviations that are favored by the militants and the opportunists of either one or both parties. However, the properties of these deviations change from one regular 4-tuple to another.

\subsubsection{An interior $\alpha$ proposal countered with a corner $\alpha$}

In this case, the party that offers a corner value for $\alpha$ always has a deviation that pleases both its opportunists and its militants. This occurs regardless of which party offers a corner solution for $\alpha$, whether it offers $\alpha=0$ or 1 , or whether the parties propose a tax or a subsidy. 


\section{Concluding remarks}

This paper has presented a political economy model to explain the low emission taxes in the U.S., and the fact that neither the Republicans and nor the Democrats advocate higher emission tax rates. The paper has two distinctive features and has arrived at two main conclusions. The first feature is its modeling of political competition which has been done à la Roemer (2001). In this setup, each party consists of two factions: one cares about the policies (militants), and the other about the probability of winning the election (opportunists). The equilibrium solution concept is the "Party Unanimity Nash Equilibrium" (PUNE). The second feature is that the model has been calibrated on the basis of the U.S. data. The voters are U.S. households as represented by the 2001 PSID survey, and the parameters of their utility function are calculated using U.S. studies.

The main result of the paper is that the PUNEs can be of two types. In one, both parties propose a very huge tax rate (combined with a budgetary rule which calls for all tax proceeds to be rebated solely on the basis of the voters' capital incomes). These tax rates correspond to the most-preferred tax rates of the militants in the Democratic and the Republican parties. If the parties offer these tax rates, the Democrats will beat the Republicans with a probability that exceeds $90 \%$.

In the other, all PUNEs entail a subsidy. This underscores the importance of distributional concerns in ensuring political support for environmental policies. Moreover, the two parties always compromise in their choices of a budgetary rule, offering an interior solution (while voters prefer the two extreme values). This underlines the importance of generating political support for environmental policies through political compromise. Under these PUNEs, the maximum winning probabilities for the two parties exceed, while their minimum winning probabilities fall below, the 50\% mark. These PUNEs appear to be more consistent with the US environmental policy which avoids high environmental taxes, and is dictated more by the concerns of the opportunists who care most about winning elections. Nevertheless, the existence of Type I PUNEs suggests the possibility of a change in this policy and arriving at an equilibrium with the militants 
at the helm. ${ }^{23}$

It will be interesting to undertake the same calibration exercise for other countries, especially those in the West, to test the robustness of our conclusions. This, however, requires quite a bit of care. The two-party political competition approach adopted here is more suited to the US, and less to the majority of European countries. ${ }^{24}$ Another extension of this analysis, would make the militants' utility endogenous by assuming that they maximize the average utility of citizens who vote for their party at equilibrium. These avenues are left for future research.

\footnotetext{
${ }^{23}$ Type II PUNEs may be considered as the more "reasonable" equilibria on two counts. First, equilibrium conditions are more stringent to satisfy for interior PUNEs (Type II) than for corner PUNEs. This is because many deviations that would be favored by both militants and opportunists are not feasible with corner solutions; they don't belong to the feasible set. Thus, if the feasible set for $\alpha$ is given by $(0,1)$ and thus open, Type I PUNEs disappear. Secondly, one can argue that in the long term the opportunists will stick to a particular party only if that party has a high enough probability to win elections. On the basis of this criterion, it is clear that Type I PUNEs are not "viable" equilibria. Under Type I PUNEs, the majority of the electorate will vote for the Left party. As noted earlier, the Left party has a probability of winning the election that varies from $90.8 \%$ to $91.2 \%$ across all possible configurations of $q_{L}$ and $q_{R}$, with an average value of $91 \%$. The $9 \%$ probability of winning is too low to keep the Right party's opportunists to stick to their party for long.

${ }^{24}$ Observe also that with the election turnout rates being much higher among the poor of Europe, the feature that all parties put relatively a higher weight on the wealthy is unlikely to apply to Europe.
} 


\section{Appendix}

Proof of Lemma 2: To prove part (i), rewrite the first-order condition for the mostpreferred level of $q$ as,

$$
\frac{\partial V(q ; m, \delta)}{\partial q}=a^{\prime}(q)+m b^{\prime}(q)+\delta\left[b^{\prime}(q) R(q)+b(q) R^{\prime}(q)\right]-\varphi^{\prime}(\bar{y}(q)) \bar{y}^{\prime}(q)=0 .
$$

Let $\widetilde{q}(\delta, m)$ denote the solution to equation (A1). We have,

$$
\frac{\partial V}{\partial q}(\widetilde{q}(\delta, m), \delta, m) \equiv 0 .
$$

Differentiating with respect to $\delta$ and $m$ yields equations (12)-(13) in the text, where $\partial^{2} V(q, \alpha, \theta) / \partial q^{2}<0$ by the concavity assumption (second-order condition). 


\section{References}

Bartels, L., 2002, Economic inequality and proportional representation, mimeo Princeton University.

Bös, D., 2000. Earmarked taxation: welfare versus political support, Journal of Public Economics 75, 439-462.

Boyer, M.and J.J. Laffont, 1998. Toward a political theory of the emergence of environmental incentive regulation, Rand Journal of Economics 30, 137-157.

Branch, E.R., 1993. Short run income elasticity of demand for residential electricity using consumer expenditure survey data, Energy Journal 14, $111-121$.

Brett, C. and M. Keen, 2000. Political uncertainty and the earmarking of environmental taxes, Journal of Public Economics 75, 315-340.

Champernowne, D.G. and F.A. Cowell, 1998. Economic Inequality and Income Distribution, Cambridge University Press.

Cremer, H., F. Gahvari and N. Ladoux, 2003. Environmental taxes with heterogeneous consumers: an application to energy consumption in France, Journal of Public Economics 87, 2791-2815.

Cremer, H., P. De Donder and F. Gahvari, 2004a. Political sustainability and the design of environmental taxes, International Tax and Public Finance 11, 703-719.

Cremer, H., P. De Donder and F. Gahvari, 2004b. Taxes, budgetary rule and majority voting, Public Choice 119, 335-358.

Cremer, H., P. De Donder and F. Gahvari, 2005. Political competition within and between parties: an application to environmental policy, Working Paper, UIUC.

Cremer, H., P. De Donder and F. Gahvari, 2007. Energy taxes in three political economy models, The B.E. Journal of Economic Analysis \& Policy, Advances, forthcoming.

Filippini, M., 1999. Swiss Residential Demand for Electricity, Applied Economic Letters 6, 533. 
Fullerton, D., 1991. Reconciling recent estimates of the marginal welfare cost of taxation, American Economic Review 81, 302-308.

Gahvari, F., 2002. Review of Ruud A. de Mooij: Environmental Taxation and the Double Dividend, Journal of Economic Literature 40, 221-223.

Gately, D. and H.G. Huntington, 2001. The Asymmetric Effects of Changes in Price and Income on Energy and Oil Demand, Economic Research Report of the C.V. Starr Center for Applied Economics, New York.

Goulder, L.H., 1995. Environmental taxation and the double dividend: a reader's guide, International Tax and Public Finance 2, 157-183.

Hodge, J.T., 1999, A cointegration analysis of U.S. energy demand elasticities, mimeo Colorado School of Mines.

Levy, G., 2004. A Model of Political Parties, Journal of Economic Theory $115,250-277$.

Lindbeck A. and J. Weibull, 1987. Balanced-budget redistribution as the outcome of political competition, Public Choice, 52, 273-297.

Marsiliani, L. and T.I. Renström, 2000. Time inconsistency in environmental policy: tax earmarking as a commitment solution, The Economic Journal 110, 123-138.

National Institute of Economics and Industry Research, 2002. The price elasticity of demand for electricity in NEM regions, Report for the National Electricity Market Management Company, Victoria, Australia.

Ninomiya, Y., 2002. Energy demand in Japan - an analysis of the past trend, Presentation at the International Workshop on Climate Policy Dialogue in Thailand, Bangkok.

Poterba, J.M., 1991. Tax policy to combat global warming: on designing a carbon tax, in R. Dornbusch and J.M. Poterba eds., Global Warming: Economic Policy Responses, The MIT Press, Cambridge, Massachusetts, $71-98$.

Roemer, J., 1999. The Democratic Political Economy of Progressive Income Taxation, Econometrica 67, 1-20. 
Roemer, J., 2001. Political Competition: Theory and Applications, Harvard University Press. 